\title{
GPR Aplicado na Localização de Armadura em Estrutura de Concreto
}

\author{
Vicente Luiz Galli ${ }^{1}$, Daniel Mariani Guirardi ${ }^{2}$, Diego Lapolli Bressan ${ }^{2}$ \\ ${ }^{1}$ Instituto de Pesquisas Tecnológicas, IPT - Seção de \\ Geotecnia \\ 2 Instituto de Pesquisas Tecnológicas, IPT - Seção de \\ Engenharia de Estruturas
}

Copyright 2014, SBGf - Sociedade Brasileira de Geofísica

Este texto foi preparado para a apresentação no VI Simpósio Brasileiro de Geofísica, Porto Alegre, 14 a 16 de outubro de 2014. Seu conteúdo foi revisado pelo Comitê Técnico do VI SimBGf, mas não necessariamente representa a opinião da SBGf ou de seus associados. É proibida a reprodução total ou parcial deste material para propósitos comerciais sem prévia autorização da SBGf.

\section{Resumo}

Este trabalho está focado na identificação de vergalhões de aço inseridos no concreto por meio do ensaio geofísico de radar. Realizou-se um levantamento na superfície de concreto de um viaduto utilizando-se antena de $1.6 \mathrm{GHz}$. Os resultados obtidos indicaram com precisão a localização da armadura de aço auxiliando na avaliação das condições estruturais em que se encontra atualmente o Viaduto mostrando a aplicabilidade e o potencial da técnica.

\section{Introdução}

O GPR tem sido usado na engenharia para identificar vergalhões e fissuras em estruturas de concreto ou então avaliar a espessura de camadas. Estas aplicações se baseiam na detecção de reflexões geradas por contrastes dielétricos existentes na interface entre os materiais.

O objetivo deste trabalho foi localizar barras de aço (vergalhões) existentes no interior de um viaduto por meio da reflexão de ondas de radar geradas na superfície a fim de auxiliar o ensaio de prova de carga do viaduto.

\section{Método}

O sistema de radar usado neste trabalho transmite pequenos pulsos de energia eletromagnética com freqüência de $1,6 \mathrm{GHz}$ usando uma antena dipolar. As ondas EM se propagam através do material e são refletidas nas interfaces com propriedades dielétricas diferentes, como as interfaces entre concreto e vergalhão. As ondas direta (Sd) e refletida (Sr) são registradas pelo sistema como sinais de amplitude em função do tempo. A antena é deslocada na superfície da estrutura e um novo pulso é enviado, repetindo o processo e, assim, uma seção dos registros da posiçãotempo é gerada. A Figura 1 ilustra o esquema de obtenção dos dados do radar.
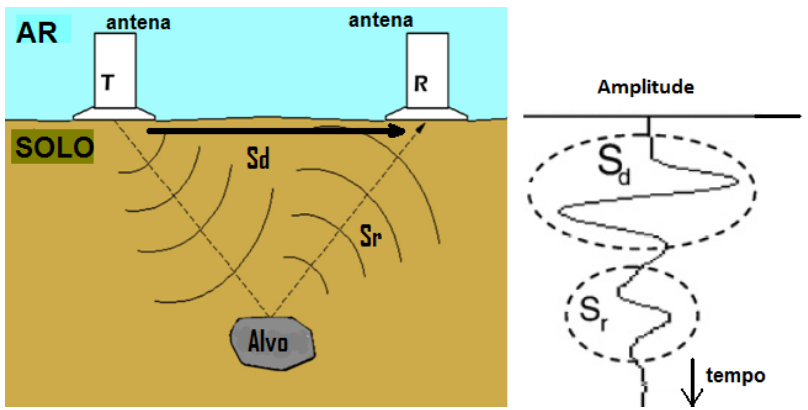

Figura 1. Registro da onda direta e da onda refletida obtido com o radar.

\section{Trabalhos realizados}

A área de estudo foi o Viaduto Aristides Bastos, na cidade de Santos-SP. Foram selecionados dois alvos, sendo um na parede lateral do viaduto e o outro na base inferior. Em cada alvo foram realizadas 36 seções de radar distribuídas em uma malha regular de $5 \mathrm{~cm} \times 5 \mathrm{~cm}$ com um registro a cada 2,5 milímetros. A Foto 1 mostra os dois alvos selecionados e a Foto 2 ilustra a aquisição dos dados na parede lateral.

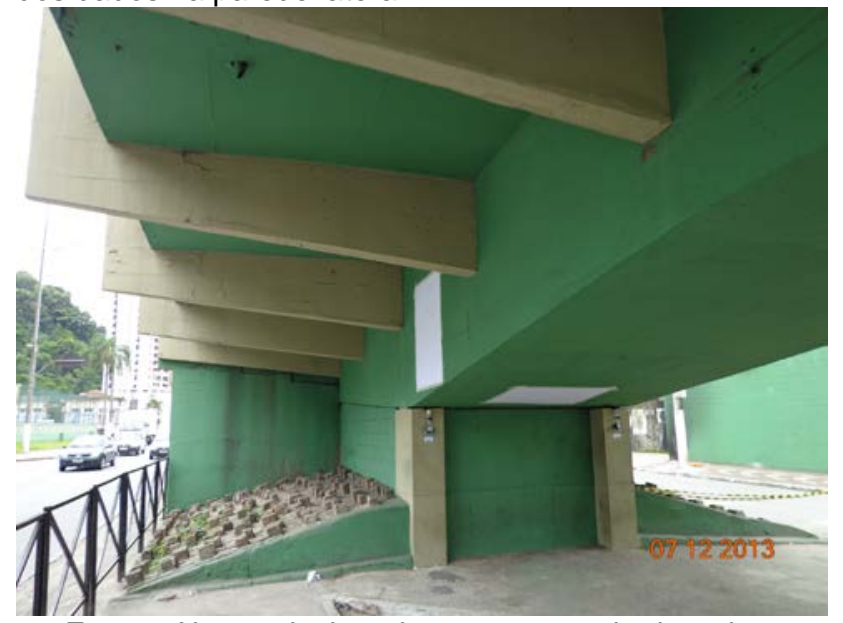

Foto 1. Alvos selecionados para o ensaio de radar. 


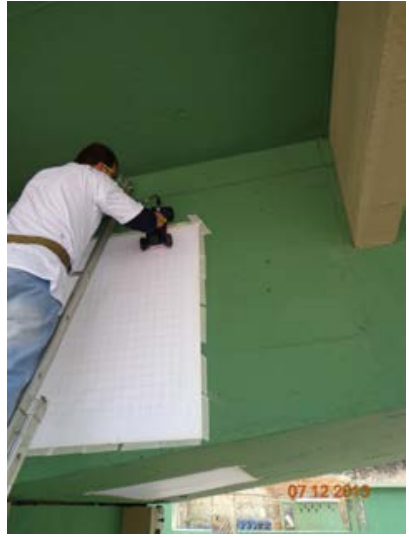

Foto 2. Ensaio de radar sobre a parede lateral do viaduto.

Os dados de radar foram coletados utilizando-se um sistema GSSI SIR-3000 com antena modelo 5100B de frequência 1,6 GHz.

\section{Resultados}

Os resultados obtidos estão sendo apresentados na forma de figuras com as seções de radar analisadas por alvo.

Alvo 1 - base inferior do viaduto

A Figura 2 ilustra uma das seções de radar com a localização em profundidade das anomalias interpretadas como sendo causadas por vergalhões. A Tabela 1 fornece as coordenadas $x, y, z$ referenciadas em relação ao gabarito fixado na superfície do concreto de cada um dos vergalhões identificados na seção ilustrada na figura 2.

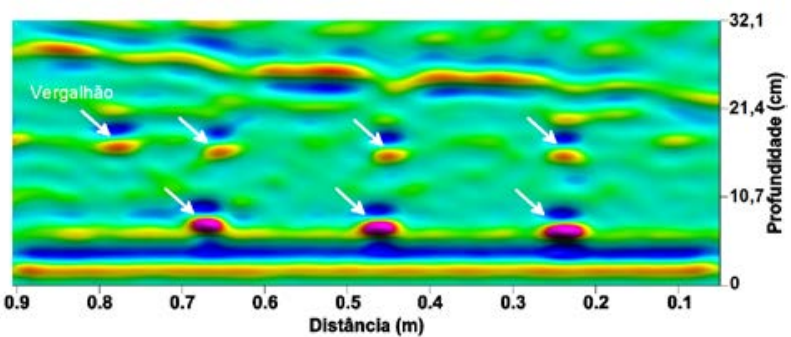

Figura 2. Uma das seções de radar processada realizada na base inferior do viaduto. As setas brancas indicam as anomalias interpretadas como sendo produzidas por vergalhões. Dados de GPR processados com Reflexw, imagem gerada no Surfer 8.

Tabela I - Coordenadas $x, y$ e $z$ dos vergalhões identificados na seção de radar ilustrada na Figura 2.

\begin{tabular}{|c|c|c|c|}
\hline Vergalhão & $\begin{array}{c}\text { Posição - } \\
\mathrm{x} \\
(\mathrm{m})\end{array}$ & $\begin{array}{c}\text { Posição - } \\
\mathrm{y}\end{array}$ & $\begin{array}{c}\text { Profundidade - } \\
(\mathrm{m})\end{array}$ \\
\hline 1 & 0,23 & 0,5 & 6,5 \\
\hline 2 & 0,46 & 0,5 & 6,9 \\
\hline 3 & 0,67 & 0,5 & 7,2 \\
\hline
\end{tabular}

\begin{tabular}{|l|l|l|l|}
\hline 4 & 0,23 & 0,5 & 15,6 \\
\hline 5 & 0,45 & 0,5 & 16,1 \\
\hline 6 & 0,65 & 0,5 & 16,8 \\
\hline 7 & 0,77 & 0,5 & 16,8 \\
\hline
\end{tabular}

Alvo 2 - parede lateral do viaduto

A Figura 3 ilustra uma das seções de radar com a localização em profundidade das anomalias interpretadas como sendo causadas por vergalhões. A Tabela 2 fornece as coordenadas $x, y, z$ referenciadas em relação ao gabarito fixado na superfície do concreto de cada um dos vergalhões identificados na seção ilustrada na figura 3.

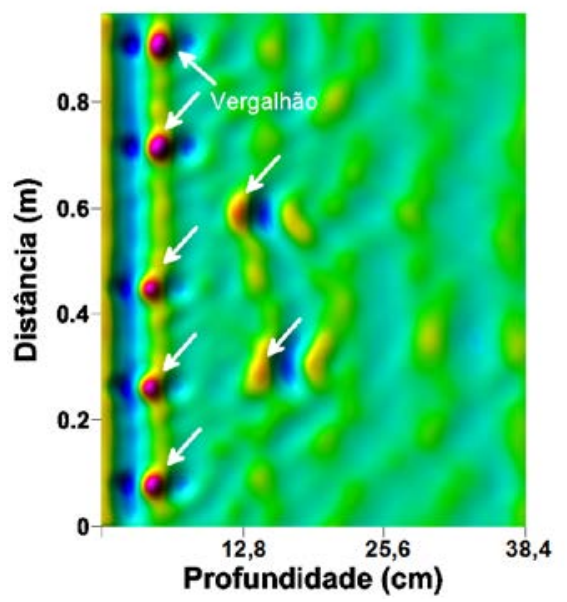

Figura 3. Uma das seções de radar processada realizada na parede lateral do viaduto. As setas brancas indicam as anomalias interpretadas como sendo produzidas por vergalhões. Dados de GPR processados com Reflexw, imagem gerada no Surfer 8 .

Tabela I - Coordenadas $x, y$ e $z$ dos vergalhões identificados na seção de radar ilustrada na Figura 3.

\begin{tabular}{|c|c|c|c|}
\hline Vergalhão & $\begin{array}{c}\text { Posição - } \\
\mathrm{x} \\
(\mathrm{m})\end{array}$ & $\begin{array}{c}\text { Posição - } \\
\mathrm{y}\end{array}$ & $\begin{array}{c}\text { Profundidade - } \\
(\mathrm{m})\end{array}$ \\
\hline 1 & 0,08 & 0,2 & 4,6 \\
\hline 2 & 0,26 & 0,2 & 4,5 \\
\hline 3 & 0,44 & 0,2 & 4,5 \\
\hline 4 & 0,71 & 0,2 & 5,2 \\
\hline 5 & 0,91 & 0,2 & 5,2 \\
\hline 6 & 0,29 & 0,2 & 14,6 \\
\hline 7 & 0,59 & 0,2 & 12,4 \\
\hline
\end{tabular}


Para visualizar toda a armadura existente sob os alvos investigados foram gerados mapas de amplitude no nível de profundidade de 0 a $0,18 \mathrm{~m}$. Os resultados são mostrados nas Figura 3 e 4.

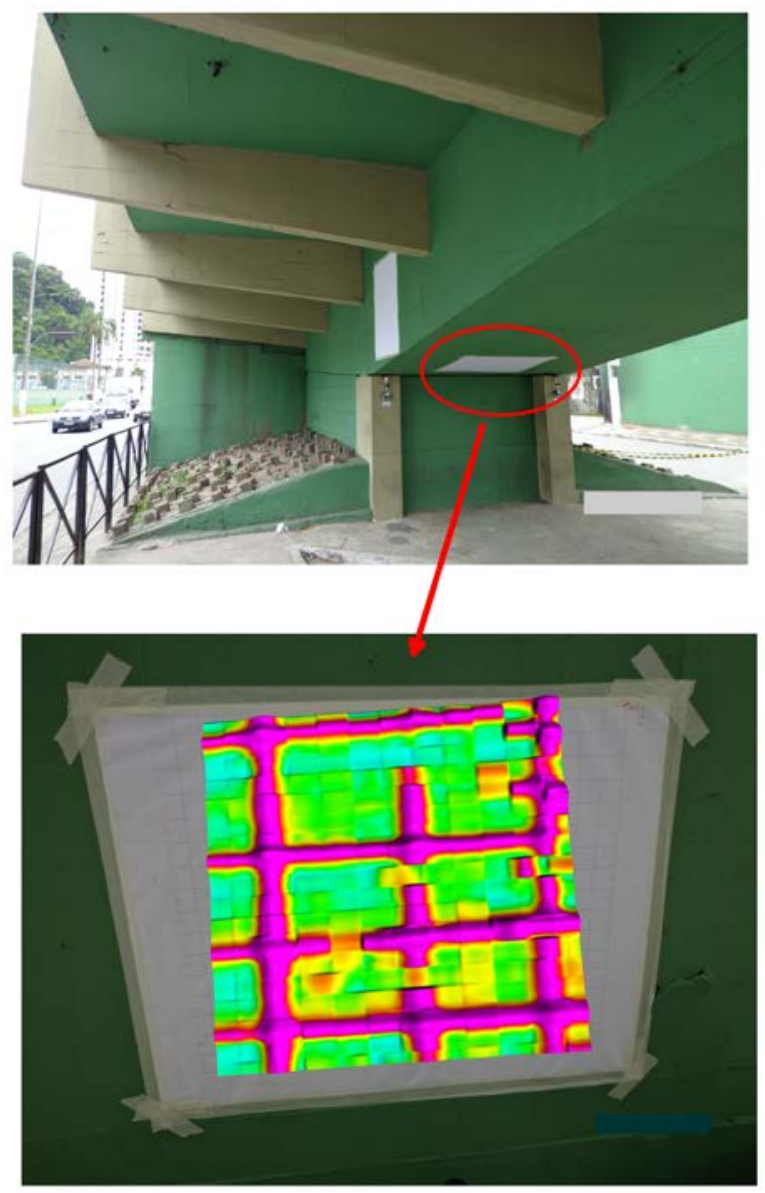

Figura 3. Montagem ilustrativa do mapa de amplitude sobreposto à base inferior do viaduto. A cor lilás reflete os vergalhões revelados pelo ensaio de radar entre 0 e $0,18 \mathrm{~m}$ de profundidade. Dados de GPR processados com Reflexw, imagem gerada no Surfer 8.

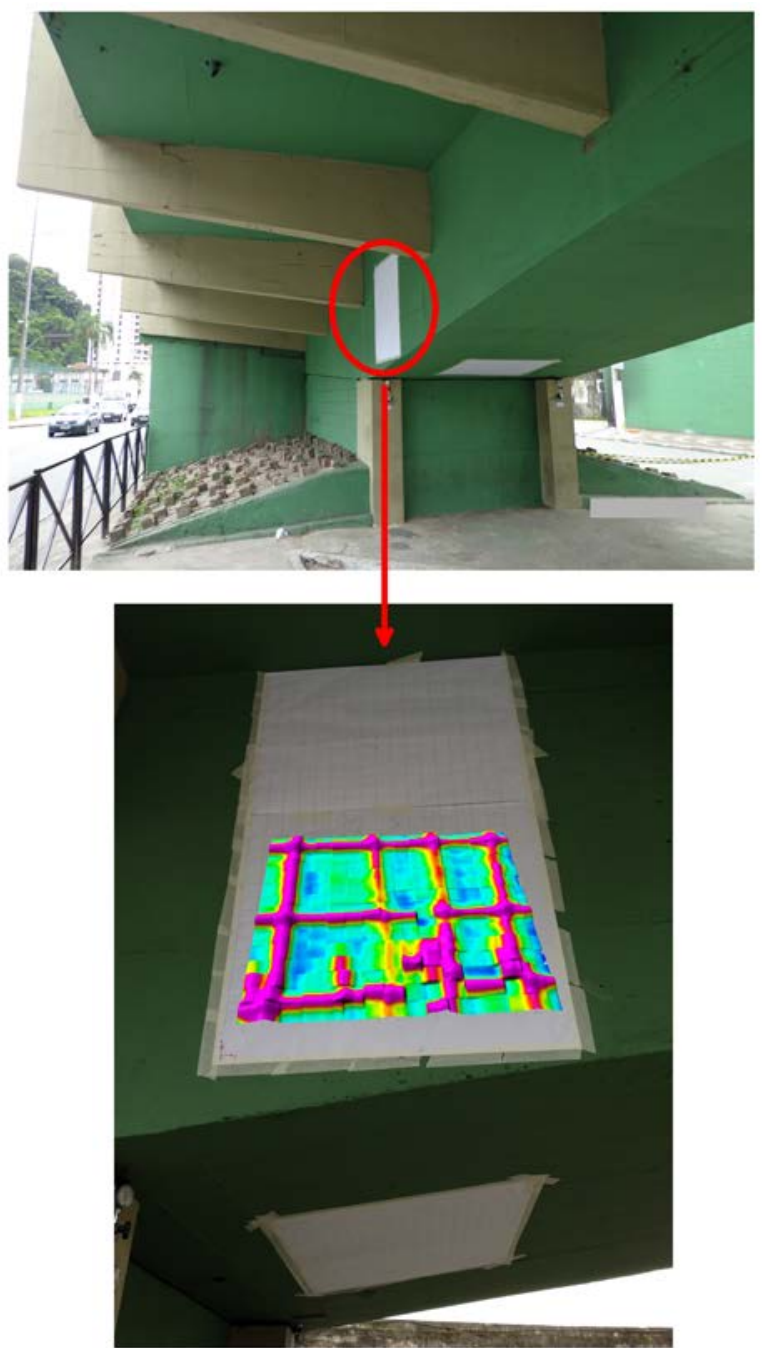

Figura 4. Montagem ilustrativa do mapa de amplitude sobreposto à parede lateral do viaduto. A cor lilás reflete os vergalhões revelados pelo ensaio de radar entre 0 e $0,18 \mathrm{~m}$ de profundidade. Dados de GPR processados com Reflexw, imagem gerada no Surfer 8

\section{Considerações Finais}

No presente trabalho foi possível, com o método de radar, identificar com precisão os vergalhões de aço existentes na estrutura de concreto do viaduto auxiliando a avaliação das condições estruturais em que se encontra atualmente o Viaduto. 


\section{Referências}

ASTM D 6432. 2005. Standard Guide for Using the Surface Ground Penetrating Radar Method for Subsurface Investigation. 17p.

Ferraro, C.C. (2003) Advanced Nondestructive Monitoring and Evaluation of Damage in Concrete Materials, Thesis of Master of Engineering, University of Florida, $294 \mathrm{p}$.

Galli, V. L, Azevedo, A. A., Sousa, L. G. (2013). Ensaio Geofísico de GPR Aplicado no Mapeamento de Fundações. 13th International Congress of the Brazilian Geophysical Society, Rio de Janeiro.

Geophysical Survey Systems, Inc - GSSI. 2011. SIR System-3000 Manual. 91p.

Sandmeier, K.J. (2008) Reflex2DQuick - Program for processing of electromagnetic reflection, refraction and transmission data - version 1.2.1. Germany.

Sauck, W. A. 1997. Radar applied to environmental problems and groundwater prospection. 5th International Congress of the Brazilian Geophysical Society, São Paulo. Short course.

Tillard, S., Dubois, J.C. (1995) Analysis of GPR Data: Wave Propagation Velocity Determination. Journal of Applied Geophysics, 33, p. 77-91. 